Gestión de la producción

Production Management 



\title{
Análisis de desempeño del área de cucharas en un proceso de sinterizado mediante el software SPSS para identificar su eficiencia dentro de una planta de sinterizado de polvos metálicos
}

\author{
Marco Antonio Díaz Martínez*, Jesús Gómez Castellanos*, Argia Lili Paz Molina*, \\ Edgar Jearvavi Vázquez Moreno*, Alma Leticia Cruz Méndez* \\ Instituto Tecnológico Superior de Pánuco. Veracruz, México.
}

Recibido: 12 de diciembre del 2017 / Aprobado: 11 de mayo del 2018

doi: 10.26439/ing.ind2018.n036.2448

RESUMEN: El presente trabajo consiste en identificar la eficiencia de un proceso de sinterizado en el área de cucharas de una planta minera. Los datos se analizaron con el software SPSS (Statistical Package for the Social Sciences), ya que esta herramienta cuenta con una flexibilidad de manejo de las variables de estudio, así como con los diferentes análisis estadísticos a los que se expusieron los datos analizados y las variables.

Palabras clave: administración de la producción - simulación por computadoras / sinterización / SPSS (programa de computadora) / eficiencia industrial / polvos metálicos

\section{Performance analysis of a sintering process ladle area using the SPSS software to identify its efficiency inside a metallic powder sinter plant}

ABstRACT: The present work involves identifying the efficiency of a sintering process ladle area of a mining plant. The data was analyzed with the SPSS (Statistical Package for the Social Sciences) software since this tool is flexible about handling the study variables, and has the different statistical analyses to which the analyzed data and variables were exposed.

Keywords: production management / computer simulation / sintering / SPSS (computer program) / industrial efficiency / metallic powders

* Correos electrónicos: marco.diaz@itspanuco.edu.mx, jesus.gomez@itspanuco. edu.mx, argia.paz@itspanuco.edu.mx, edgar.vazquez@itspanuco.edu.mx, alma. cruz@itspanuco.edu.mx 


\section{INTRODUCCIÓN}

La pulvimetalurgia es un proceso de suma importancia, ya que su objetivo es cumplir con las especificaciones requeridas por una empresa dedicada al tratamiento de materiales mediante fundición. Dentro de una planta donde se lleva a cabo el proceso de sinterizado, su función es generar recubrimientos de material o la generación de materia prima para enviar a otra empresa que se dedicará a crear piezas mecánicas o una transformación con esta materia prima obtenida del proceso de sinterizado (manganeso, cobre, plomo, estaño, etc.), junto con la rebaba obtenida del proceso donde se remueve el excedente de la aleación que recibe el nombre de cepillado final y polvo de la misma aleación a fabricar.

La pulvimetalurgia o metalurgia de polvos es el proceso donde los metales en su estado inicial de polvo se compactan mediante el proceso de sinterizado para darles formas deseadas complejas y son calentadas hasta una temperatura por debajo del punto de fusión para formar una pieza sólida (Kalpakjia, 2008).

La fabricación de elementos y materiales se inicia con una mezcla de polvos metálicos, cerámicos o mezcla de ambos elementos con el lubricante y los aditivos. Esta mezcla se compacta dentro de un molde mediante la aplicación de presión y, después de este proceso, pasa al proceso de sinterizado como etapa final (Randall, 1989).

Luego de realizado el proceso de sinterizado, varias piezas son utilizadas puramente de acuerdo con sus propiedades mecánicas, como plasticidad, fragilidad, dureza, maleabilidad, etc., mientras que otras son expuestas a operaciones de acabado como mecanizado, calibrado, recubrimientos o tratamientos térmicos como nitruración, temple, entre otros.

En este trabajo se realizará un análisis completo del desempeño de los operadores que intervienen en el área de producción de cucharas en un proceso de sinterizado y de los resultados obtenidos en cada uno de los turnos de trabajo, con lo cual se determinará la eficiencia del área de las cucharas y el impacto que tiene con respecto al proceso completo de sinterización.

\subsection{Proceso de sinterizado y proceso de cucharas}

La metalurgia de polvos es una competencia muy importante con respecto a los procesos de fundición, forjado y maquinado o mecanizado, 
en particular para partes relativamente complejas fabricadas con aleaciones de alta resistencia (Kalpakjia, 2008).

Los metales más utilizados en la metalurgia de polvos son hierro, cobre, aluminio, estaño, níquel, titanio y los metales refractarios, para las partes fabricadas en el proceso de sinterizado que es el último proceso.

En el proceso de cucharas, el cual se realiza dentro del proceso de sinterizado del material, donde las cucharas son unos contenedores cuya función es vaciar la mezcla que viene de las tolvas e inician la reacción de la mezcla, luego de haberse quemado el material, se procede al vaciado del material hacia los patios donde los trascabos transportan el material sinterizado al proceso de fundición.

\subsection{Manufactura del polvo y sus características}

La manufactura del polvo es muy importante y se debe trabajar bajo ciertas especificaciones, las cuales determinan ciertas características como las propiedades físicas y mecánicas de compactación. Estas especificaciones incluyen requerimientos sobre el tamaño, la forma y las estructuras de la partícula, y las áreas de las superficies.

\subsubsection{Tamaño de partículas y su distribución}

Se refiere a las dimensiones de los polvos donde las partículas se separan haciéndolas pasar a través de una serie de cribas de tamaños menores de malla. Los polvos se colocan sobre una criba de un cierto número de mallas y esta vibra para que las partículas pequeñas que caben en las aberturas caigan a la próxima criba.

Cuando las aberturas en la criba son menores que el recíproco del número de malla debido al espesor del alambre de la criba, se tiene que utilizar la siguiente formula (Groover, 2007).

$$
P S=\frac{1}{M C}-t_{w}
$$

Donde $P S=$ Tamaño de partícula, $M C=$ Número de malla, $t_{w},=$ Grueso del alambre. 
Tamaño de partícula que no pasa a través de la malla

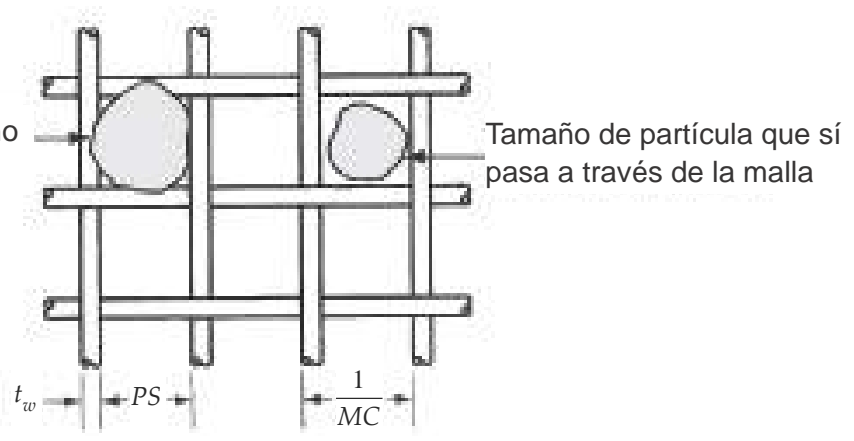

Figura 1. Malla de criba para seleccionar tamaños de partícula Fuente: Groover (2007)

\subsubsection{Forma y estructura interna de las partículas}

En la siguiente figura, se muestran diferentes formas de polvos metálicos que pueden catalogarse en varios tipos. En estos tipos existen diferencias, tanto en forma como en tamaño; sin embargo, es importante mencionar que una manera útil de medir la forma es el aspecto, así como la dimensión máxima y la mínima de una partícula dada (Groover, 2007).

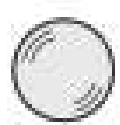

Esférica

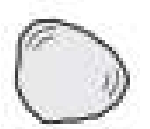

Redondeada

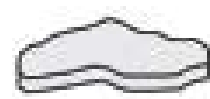

Hojuela

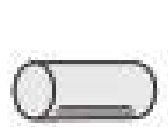

Cilíndrica Esponjosa

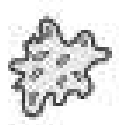

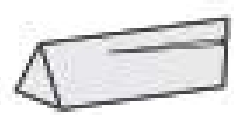

Acicular

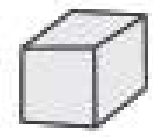

Cúbica

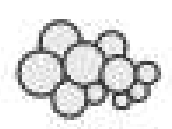

Agregada

Figura 2. Formas ideales de partículas de metalurgia de polvos Fuente: Groover (2007)

Para las partículas esféricas, tienen una razón de 1,0; en el caso de los granos aciculares, pueden ser de 2,0 a 4,0. Cabe mencionar que es de suma importancia conocer técnicas microscópicas para determinar las características de las formas. 


\subsection{3 Área superficial}

En cuanto a cómo determinar el área superficial suponiendo que la forma sea una esfera totalmente, su área A y su volumen $\mathrm{V}$ están dados por:

$$
\begin{aligned}
& A=\pi D^{2} \\
& V=\frac{\pi D^{3}}{6}
\end{aligned}
$$

donde $\mathrm{D}$ = diámetro de la partícula esférica, $\mathrm{mm}$ (in). La razón de área a volumen $\mathrm{A} / \mathrm{V}$ para una esfera se determina entonces por:

$$
\frac{A}{V}=\frac{6}{D}
$$

En general, la razón del área del volumen puede expresarse para cualquier forma como:

$$
\frac{A}{V}=\frac{K}{D}
$$

donde $K$ es el factor de forma y $D$ es igual al diámetro de una esfera de volumen equivalente al de una partícula no esférica, mm (in) (Groover, 2007).

\section{REVISIÓN LITERARIA}

A continuación, se mencionan algunas aportaciones de investigaciones que hacen referencia a las características, aplicaciones, comportamientos o influencias que tiene el proceso de sinterizado.

La simulación del proceso de compactación en molde de polvo metálico para la elaboración de una pieza pulvimetalúrgica, donde aplica el modelo de plasticidad modificado, es resuelta mediante el uso de softwares de cálculo como Abus. El resultado permite que el movimiento de punzones optimice la calidad de la pieza acabada; asimismo, se obtiene una distribución de tensiones en distintas zonas del molde (M. Prado, D. Riera, 1998).

Las nuevas demandas y solicitudes en el sector automotor están haciendo que se potencialicen los esfuerzos en aumentar las facilidades y reducir los costos de aceros sinterizados. Las propiedades finales de los componentes sinterizados y su costo dependen de dos factores principales: la densidad y el sistema de aleación (Campos, 2008). 
La pulvimetalurgia es una tecnología bien conocida por el objetivo de producir piezas de forma amplia a bajo costo, pero constantemente con una pérdida de propiedades mecánicas si se la compara con tecnologías alternativas para obtener las mismas piezas o los mismos resultados. Sin embargo, mediante esta tecnología, también se pueden generar y obtener piezas de alta disponibilidad, de acuerdo con la ruta de procesado y el nivel de porosidad (Torralba, 2014).

Otra modalidad es la del prensado en caliente, en la que la masa de polvo sometida a compresión se calienta simultáneamente por el paso de una corriente de elevada intensidad (Montes, 2003).

\section{METODOLOGÍA}

\subsection{Metodología para producción de polvos}

Existen varios métodos para poder producir polvos metálicos. La elección depende de los requisitos del producto final y de las operaciones que intervienen en el proceso de metalurgia, como producción de polvos, mezclas, compactación, sinterización y operaciones de acabado.

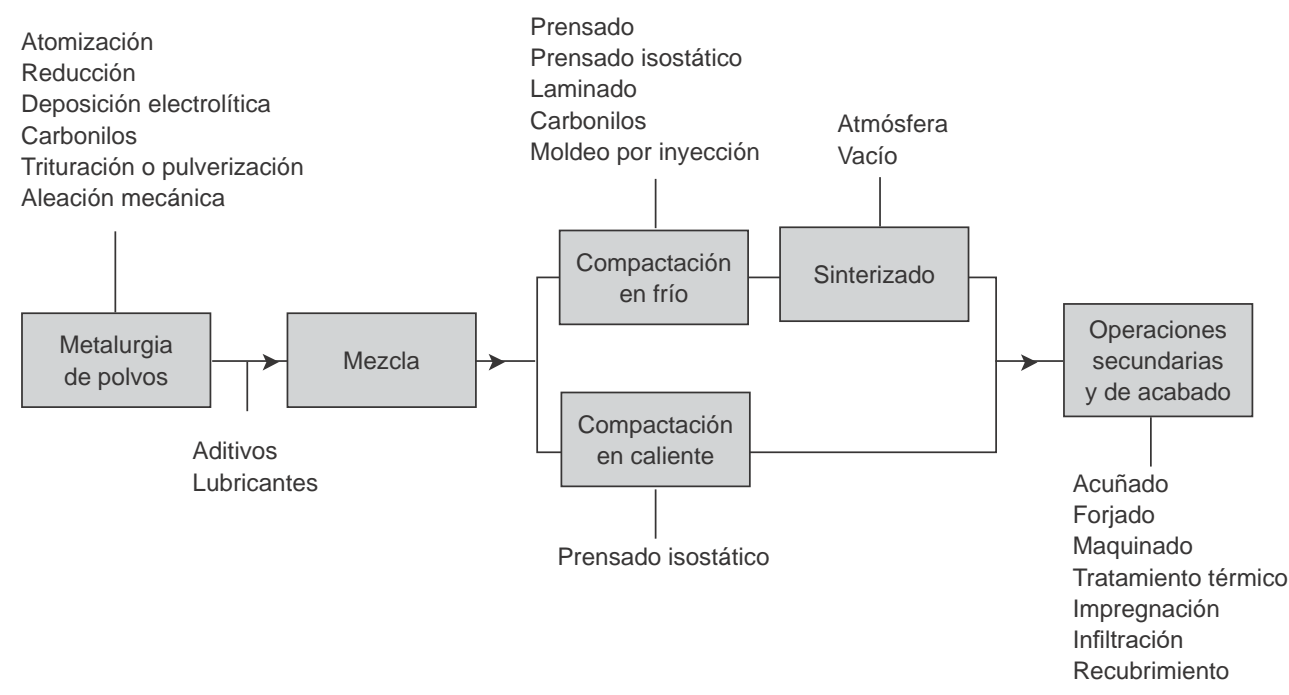

Figura 3. Procesos y operaciones comprendidas en la fabricación de partes mediante metalurgia de polvos

Fuente: Kalpakjian (2008) 
El proceso de sinterizado es una operación de tratamiento térmico que se ejecuta mediante una compactación para unir partículas metálicas, lo cual genera un aumento en su fuerza y resistencia. Este proceso y tratamiento se lleva a cabo a temperaturas entre 0,6 y 0,8 del punto fusión del metal. La fuerza básica que mueve el sinterizado es la reducción de la energía superficial (De Garmo, 2003).

En la actualidad, el proceso de sinterizado controla la atmósfera del horno que se va a trabajar, y el objetivo de tener controlada la atmósfera del horno es proteger la oxidación del material, proporcionar una reducción en la atmósfera para remover los óxidos existentes, y suministrar un ambiente carburizado para ayudar a la remoción de lubricantes y aglutinantes que son utilizados en el proceso de prensado.

La siguiente figura muestra, en una escala microscópica, los cambios que suceden durante el proceso de sinterizado de los polvos metálicos. El sinterizado implica el transporte de masa para la creación de cuellos de botella y transformarlos en granos.

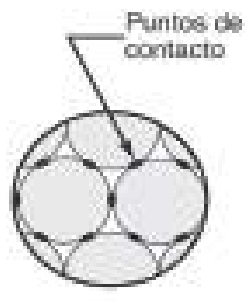

1)

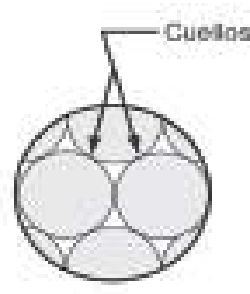

27

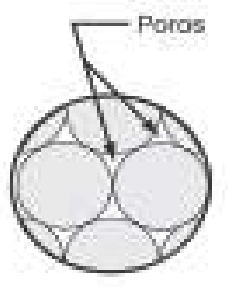

3)

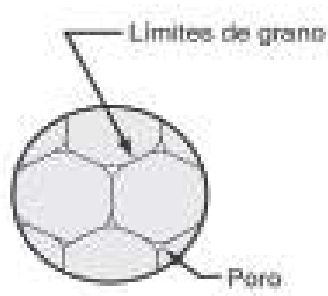

4)

Figura 4. Escala microscópica de sinterizado: 1) la unión de las partículas se inicia en los puntos de contacto, 2) los puntos crecen para convertirse en cuellos de botella, 3) los poros se reducen de tamaño, y 4) se desarrollan límites de granos entre partículas Fuente: Groover (2007)

\subsection{Descripción del método}

Para la realización del análisis del proceso, se tomaron muestras de desempeño en diferentes turnos de trabajo y se analizaron los tiempos muertos, tiempo de productivo directo, tiempo de espera en proceso, espera de material y mantenimiento. 
Asimismo, se realizó un modelado con el software Flexsim del proceso completo de sinterizado, donde se mostraron cada uno de los elementos y las operaciones que intervienen en el proceso de sinterizado. Esto ayudó a poder entender la distribución de las materias primas y las dependencias entre procesos, así como la cantidad de operadores o supervisores que intervienen en el sistema (Beaverstock, 2011).

Los resultados del análisis de desempeño, así como los comparativos en cada turno de trabajo, se realizarán en el software de SPSS, ya que lo que se busca no solamente es analizar los datos sino también implementar nuevas tecnologías con el fin de ir evolucionando a una cultura de pensamiento de la industria 4.0.

En la siguiente figura, se puede visualizar el modelo de simulación de un proceso de sinterizado hecho con el software Flexsim, ya que servirá como apoyo para poder tener un mejor control de las operaciones que intervienen en todo el sistema y, sobre todo, ir impulsando el uso de nuevas tecnologías para la mejora continua del proceso de sinterizado en tiempo real.

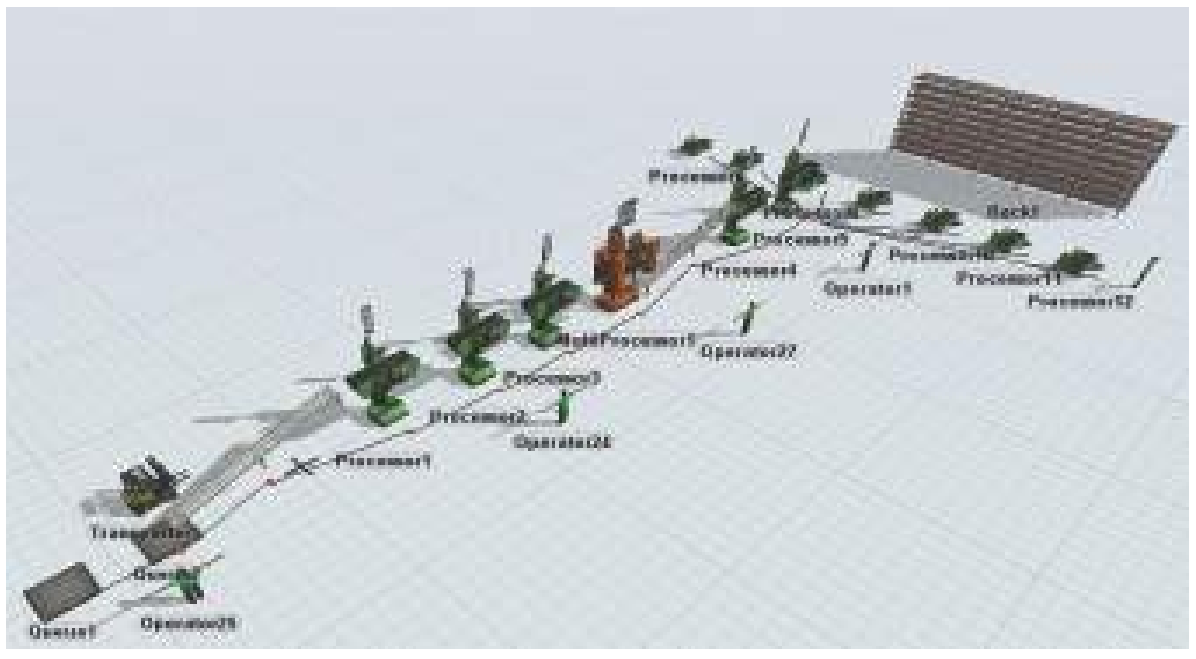

Figura 5. Modelado en Flexsim del proceso de sinterizado Elaboración propia 


\subsection{Descripción de actividades por área}

Tabla 1

Descripción de las actividades que intervienen previamente al proceso mismo de sinterizado

\begin{tabular}{|c|c|}
\hline Área & Descripción de actividades \\
\hline 1. Área de conos de materia prima & $\begin{array}{l}\text { Se necesita de un trascabo exclusivo para hacer } \\
\text { las mezclas en el tercer turno únicamente y se tra- } \\
\text { baja aproximadamente } 6 \text { horas efectivas para for- } \\
\text { mar } 5 \text { o } 7 \text { conos para los } 3 \text { turnos. Las prioridades } \\
\text { son: desalojar los patios, llenar tolvas de recibo } \\
\text { cuando esté por terminarse el material. }\end{array}$ \\
\hline 2. Tolva de recibo & $\begin{array}{l}\text { Trabajan de } 30 \text { a } 40 \text { minutos en promedio para } \\
\text { llenar la tolva de materia prima. Amplían la capa- } \\
\text { cidad de la tolva a } 60 T \text {. Estas tolvas usan un yucle } \\
\text { para acercar la mezcla a la tolva de recibo y así } \\
\text { evitar largos recorridos del trascabo y la pérdida } \\
\text { del material en el camino. }\end{array}$ \\
\hline 3. Tolvas de materia prima & $\begin{array}{l}\text { Trabajan al } 30 \% \text { o } 40 \% \text { de su capacidad, que son } \\
\text { de } 90 \text { a } 120 \text { minutos, para llenar todas las tolvas. } \\
\text { Una vez llenas, deben alcanzar para trabajar entre } \\
4 \text { y } 5 \text { horas continuas y volver a reabastecerse. }\end{array}$ \\
\hline 4. Kan ban & $\begin{array}{l}\text { La capacidad a la que trabajan es a } 60 \text { tonela- } \\
\text { das/hora y } 450 \text { toneladas/turno } 100 \% \text { y } 360 \text { tone- } \\
\text { ladas/turno } 80 \% \text {. }\end{array}$ \\
\hline 5. Mezclador & $\begin{array}{l}\text { Tiene una capacidad de } 60 \text { toneladas/hora, } 450 \\
\text { toneladas/turno } 100 \% \text { y } 380 \text { toneladas/turno } 80 \%\end{array}$ \\
\hline 6. Tolvas de día & $\begin{array}{l}\text { Tienen capacidad de } 20 \text { toneladas c/u a } 30 \mathrm{~m} \text { o } 40 \\
\text { toneladas c/u para tener una capacidad de } 60 \text { u } 80 \\
\text { toneladas en una hora. }\end{array}$ \\
\hline 7. Cucharas & $\begin{array}{l}\text { Transportan todas las demás operaciones, y esto } \\
\text { significa que esta operación no debe tener desa- } \\
\text { bastecimiento de material. }\end{array}$ \\
\hline
\end{tabular}

Elaboración propia 


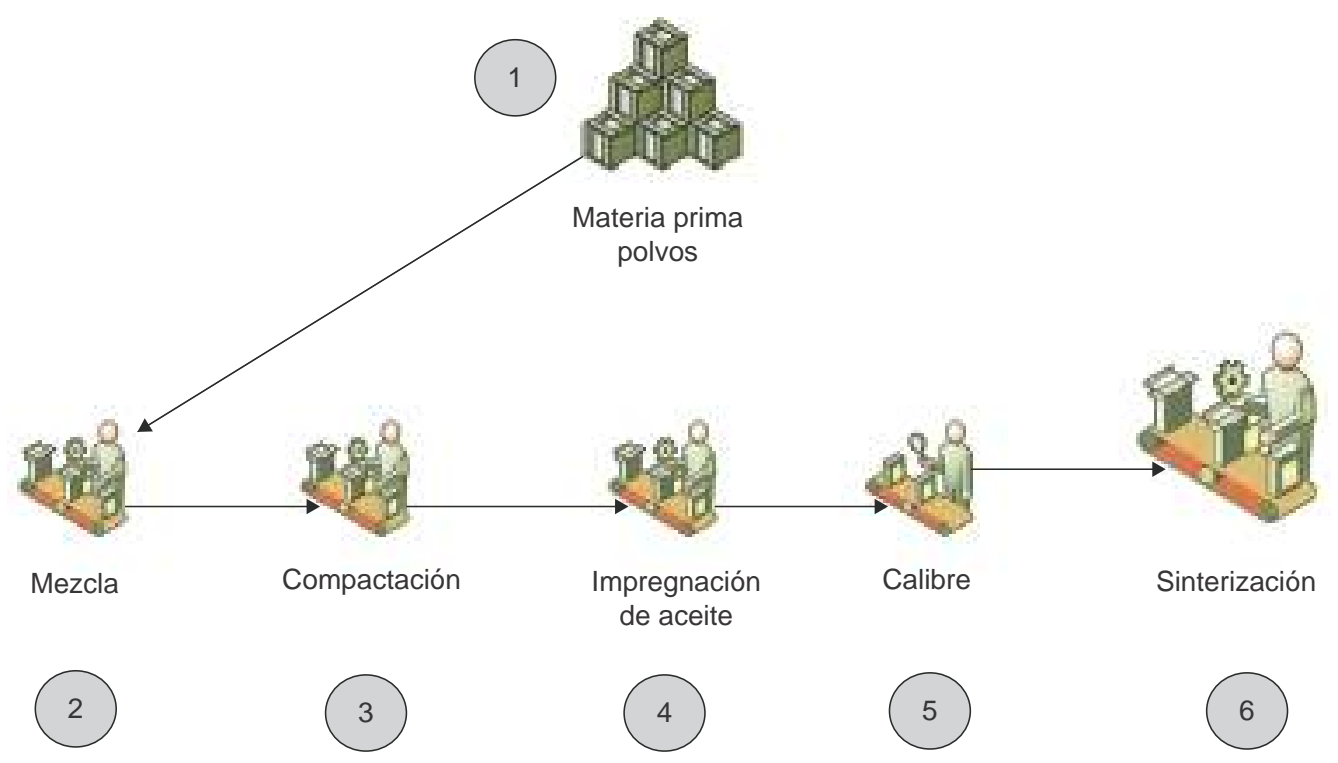

Figura 6. Áreas del proceso de sinterizado Elaboración propia

En este diagrama se puede visualizar la existencia de las operaciones que intervienen en el proceso de sinterizado y cómo este proceso es el último y más importante, ya que de este proceso dependerá la intervención de muchas empresas en la compra de la materia prima para la creación de piezas mecánicas y de nuevos elementos de sujeción.

\section{RESULTADOS}

En las siguientes tablas se muestran los resultados del análisis por día de las cucharas y los resultados de los diferentes tiempos que se obtuvieron, como tiempo muerto (TM), tiempo productivo directo (TPD), tiempo de espera (TE), tiempo de alimentación del personal (TAP) y total (T). Los análisis fueron desarrollados en el software de estadística SPSS y la técnica que se utilizó para la toma de tiempos fue la de muestreo del trabajo al considerar una muestra de 12 días y realizar 36 observaciones completas en tres turnos de trabajo. 
De acuerdo con los resultados obtenidos en el análisis de desempeño del primer turno (tabla 2), se puede ver que el porcentaje más alto es el tiempo productivo directo con un 53,85 \% y el siguiente es el tiempo de espera (TE) con un 32,19\%. Este a su vez se analiza al encontrar tres fuentes principales (tabla 3). Se puede notar que el de mayor impacto es el tiempo de espera de proceso con un $76 \%$ del total $(32,19 \%)$. De acuerdo con el primer turno analizado, hay un alto porcentaje de espera de proceso.

De acuerdo con los resultados obtenidos en el análisis de desempeño del segundo turno (tabla 4), se puede ver que el porcentaje más alto es el tiempo productivo directo con un 54,27 \% que, en comparación con el primer turno, es ligeramente mayor, y que los tiempos de espera representan el segundo tiempo más elevado con 29,18\%. Al analizarlos, se observa que se debe a tres fuentes principales (tabla 5), y es el tiempo de espera de proceso el de mayor impacto con un $83,16 \%$ del total $(29,18 \%)$. De acuerdo con el segundo turno analizado, se ve nuevamente que hay un alto porcentaje de espera de proceso.

Los tiempos de espera se generan principalmente por falta de material (en tolvas), mantenimientos por fallas en equipos o bandas y por falta del camión fuera de carretera o cargador frontal.

Según los resultados obtenidos en el análisis de desempeño del tercer turno, se puede ver que el porcentaje más alto es el tiempo productivo directo con un 50,85\% que, en comparación con el segundo turno, bajó un $3 \%$, y que los tiempos de espera representan el segundo tiempo más elevado con 33,54 \%. Al analizarlos, encontramos que son dos las fuentes principales (tabla 7). El tiempo de espera de proceso es el de mayor impacto con un $84,42 \%$ del total (33,54\%), y se nota que el tercer turno tiene el tiempo más alto de espera. Al comparar los tres turnos es observable que el tercer turno es el menos productivo y sus tiempos de espera más altos son en proceso, lo que indica una mayor falta de control y/o supervisión en las operaciones. En el caso de la actividad de mantenimiento, se presenta con un $0 \%$ debido a que en este turno no se tiene personal de mantenimiento asignado y, en caso de que existiera alguna urgencia, se solicita apoyo a mantenimiento de otras áreas. 


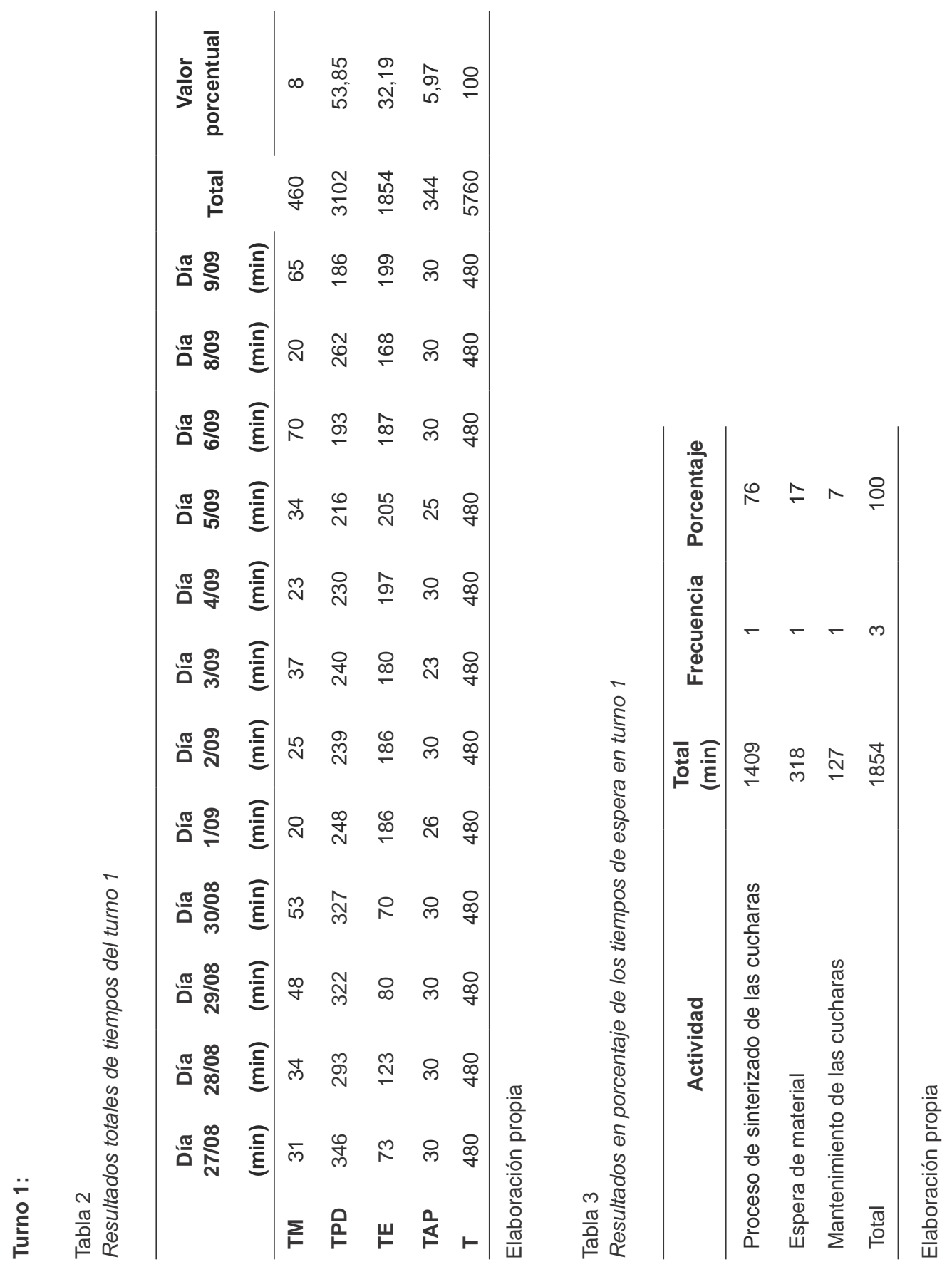




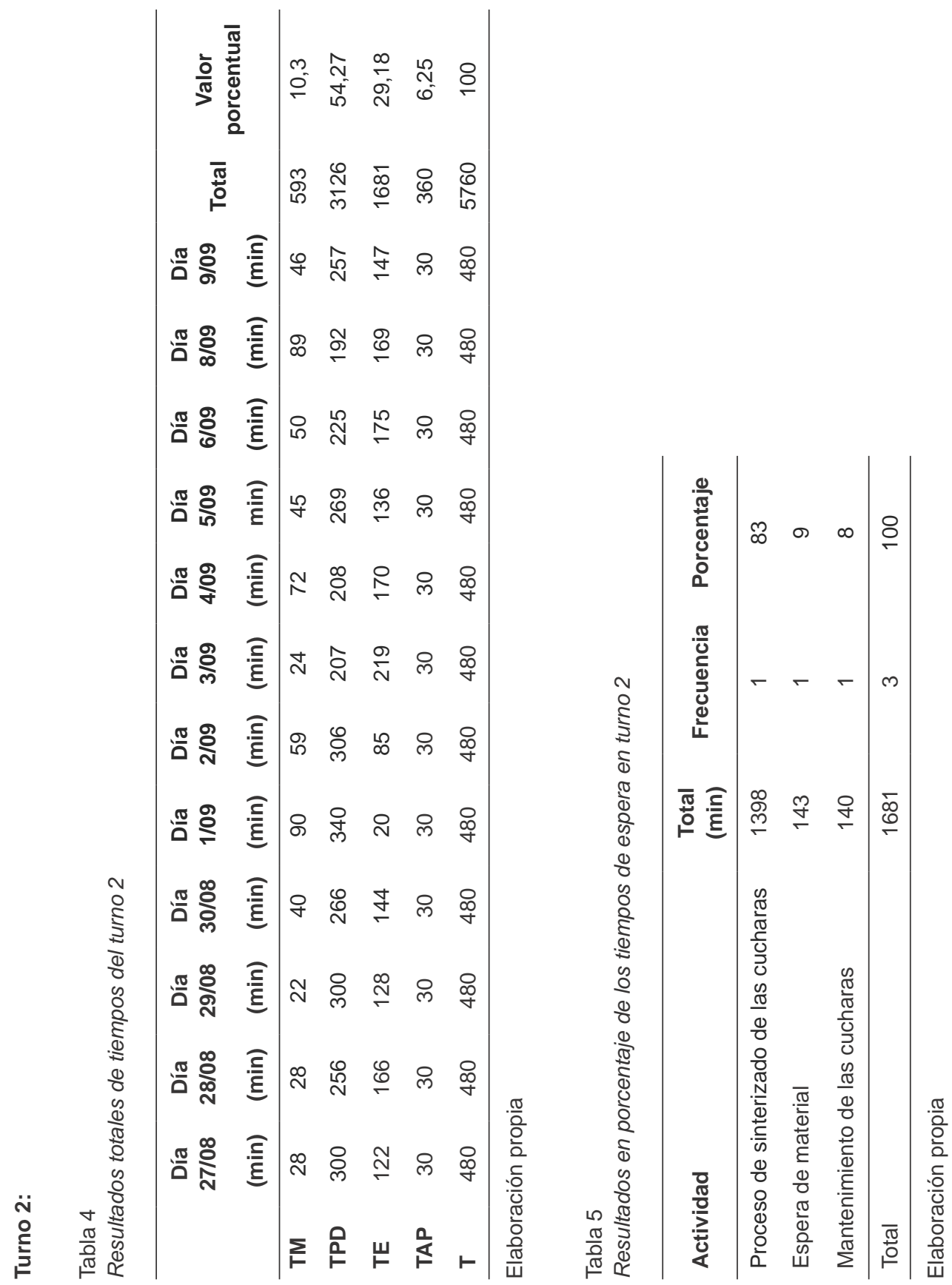




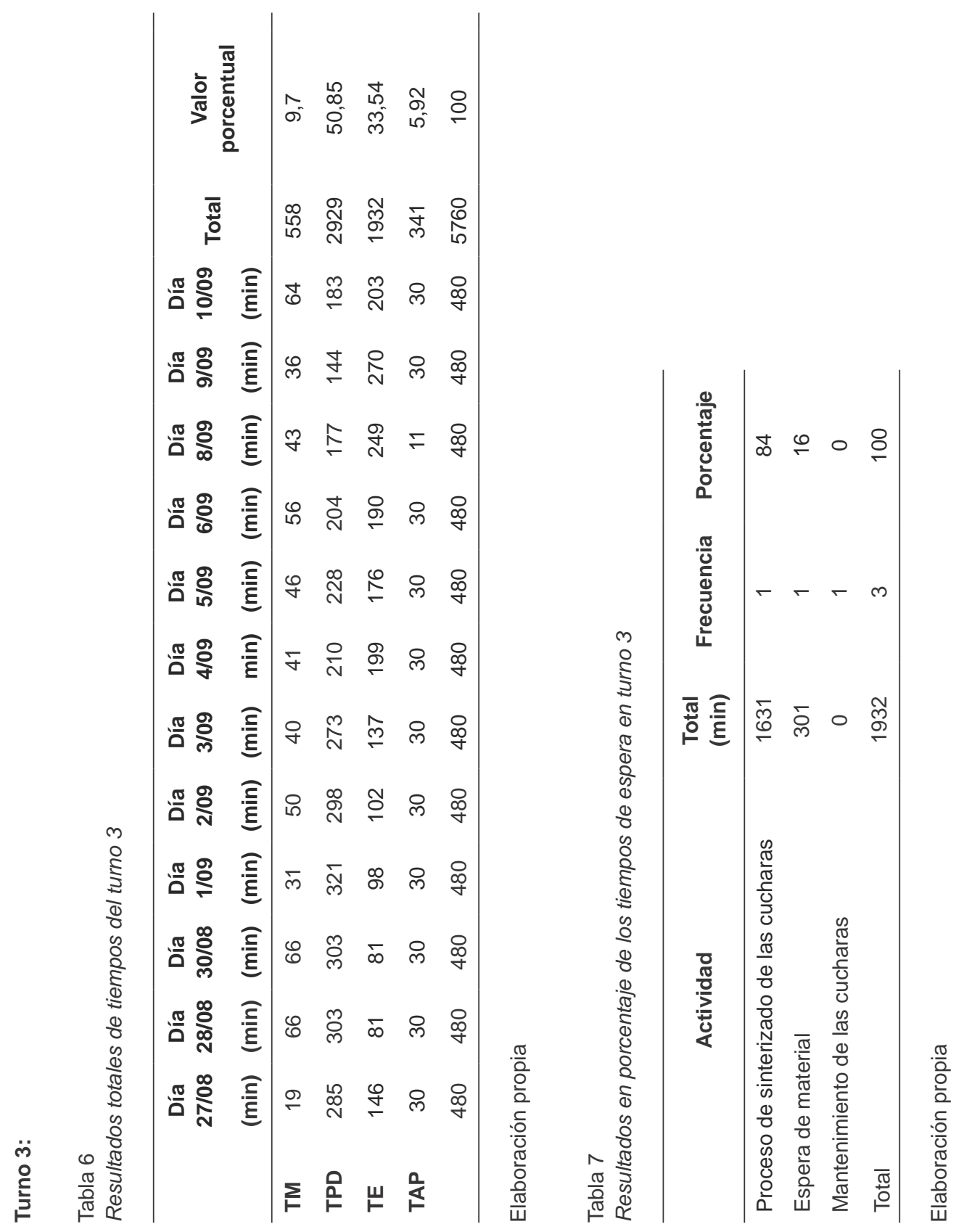


De acuerdo con los resultados de los tiempos de los tres turnos, se aplicó una distribución estadística de Erlang en percentiles para observar la separación que puede haber en los resultados de cada uno de los turnos analizados. A continuación, se muestra la cantidad de datos válidos analizados $(\mathrm{N})$.

Tabla 8

Resultados de tiempos máximos y mínimos

\begin{tabular}{lcccc}
\hline & & Turno 1 & Turno 2 & Turno 3 \\
\hline N & Válido & 4 & 4 & 4 \\
Percentiles & 25 & 373 & 418,25 & 395,25 \\
& 50 & 1157 & 1137 & 1245 \\
& 75 & 2790 & 2764,75 & 2679,75 \\
\hline
\end{tabular}

Elaboración propia

Tabla 9

Distribución de frecuencias de los tiempos

\begin{tabular}{l|rrr}
\hline \multicolumn{1}{c|}{ Actividad } & $\begin{array}{r}\text { Total } \\
(\mathbf{m i n})\end{array}$ & Frecuencia & Porcentaje \\
\hline \multicolumn{1}{c}{ Turno 1 } & & & \\
Tiempos muertos & 360 & 1 & 8 \\
Tiempo de producto directo & 1854 & 1 & 32 \\
Tiempo de espera & 344 & 1 & 6 \\
Tiempo de alimentación personal & 5760 & 4 & 100 \\
Total & & & \\
\hline \multicolumn{1}{c}{ Turno 2 } & 593 & 1 & 10 \\
Tiempos muertos & 3126 & 1 & 54 \\
Tiempo de producto directo & 1681 & 1 & 29 \\
Tiempo de espera & 360 & 1 & 6 \\
Tiempo de alimentación personal & 5760 & 4 & 100 \\
Total & & & 10 \\
\hline \multicolumn{1}{c}{ Turno 3 } & 558 & 1 & 51 \\
Tiempos muertos & 2929 & 1 & 34 \\
Tiempo de producto directo & 1932 & 1 & 6 \\
Tiempo de espera & 341 & 1 & 100 \\
Tiempo de alimentación personal & 5760 & 4 & \\
Total & & & \\
\hline
\end{tabular}

Elaboración propia 


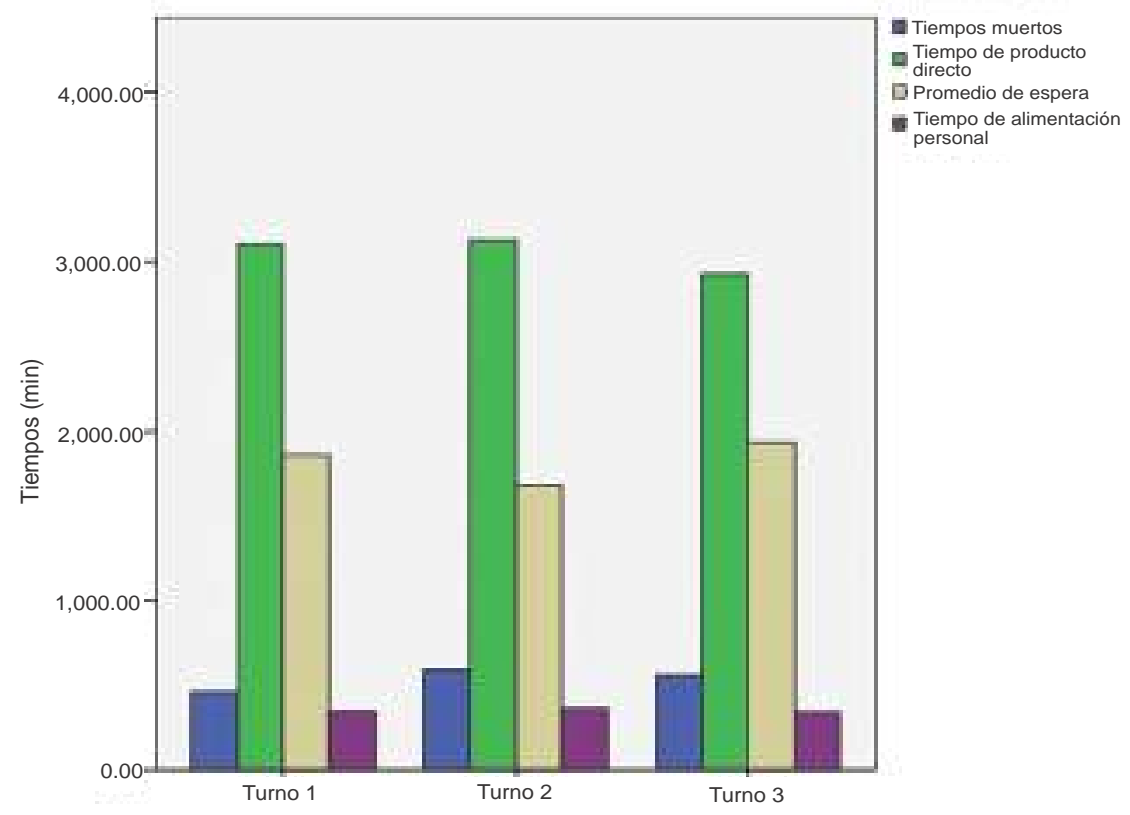

Figura 7. Resultados comparativos de los tiempos máximos y mínimos de los tres turnos de trabajo

Elaboración propia

Según los resultados de la figura 7 , se puede observar que en los tiempos muertos existe una diferencia de $2 \%$ entre los turnos 2 y 3 con respecto al turno 1 , que tiene un $8 \%$ que sería el tiempo más óptimo.

En los tiempos de producto directo se observa que los turnos 1 y 2 tienen un $54 \%$ y el turno 3 un $51 \%$, que sería el tiempo menos productivo y, por último, en los tiempos de espera existe una variación notable donde el turno 2 tiene un $29 \%$, que es el más bajo; el turno 1 tiene un $32 \%$ y el turno 3 , un $34 \%$, que es el más alto. 
4.1 Análisis de resultados de los tiempos de vaciado en el proceso de cucharas (Módulo 1)

Tabla 10

Resultados del análisis del tiempo de vaciado del proceso de cucharas en el turno 1

Cucharas Vaciadas $\begin{gathered}\text { Turno } \\ \text { anterior Tiempos por cuchara }(\mathrm{min}) \quad \text { Suma Promedio }\end{gathered}$

\begin{tabular}{ccccccccccc}
\hline 1 & 7 & 16 & 42 & 40 & 42 & 57 & 42 & 41 & 264 & $44,0^{*}$ \\
2 & 6 & 37 & 66 & 67 & 55 & 54 & 66 & & 308 & 61,6 \\
3 & 5 & 57 & 78 & 99 & 89 & 70 & & & 336 & 84, \\
\hline Total & 18 & & & & & & & & & \\
\hline
\end{tabular}

*Proceso con mayor eficiencia observada.

Elaboración propia

Tabla 11

Resultados del análisis del tiempo de vaciado del proceso de cucharas en el turno 2

Cucharas Vaciadas $\begin{gathered}\text { Turno } \\ \text { anterior }\end{gathered}$ Tiempos por cuchara (min) Suma Promedio

\begin{tabular}{ccccccccccc}
\hline 1 & 7 & 14 & 49 & 47 & 68 & 46 & 48 & 48 & 306 & $51,0^{*}$ \\
2 & 5 & 17 & 64 & 50 & 70 & 71 & & & 255 & 63,75 \\
3 & 5 & 19 & 64 & 65 & 69 & 73 & & & 290 & 72,5 \\
\hline Total & 17 & & & & & & & & & \\
\hline
\end{tabular}

* Proceso con mayor eficiencia observada.

Elaboración propia

Tabla 12

Resultados del análisis del tiempo de vaciado del proceso de cucharas en el turno 3

\begin{tabular}{cccccccccccc}
\hline Cucharas & Vaciadas & $\begin{array}{c}\text { Turno } \\
\text { anterior }\end{array}$ & \multicolumn{1}{c}{ Tiempos por cuchara (min) } & Suma & Promedio \\
\hline 1 & 7 & 11 & 60 & 33 & 114 & 35 & 49 & 35 & 326 & $54,33^{*}$ \\
2 & 6 & 11 & 54 & 57 & 80 & 59 & 97 & 347 & 69,4 \\
3 & 4 & 11 & 80 & 57 & 65 & & & & 202 & 67,33 \\
\hline Total & 17 & & & & & & & & & &
\end{tabular}

* Proceso con mayor eficiencia observada.

Elaboración propia 
4.2 Análisis de resultados de los tiempos de vaciado en el proceso de cucharas (Módulo 2)

Tabla 13

Resultados del análisis del tiempo de vaciado del proceso de cucharas en el turno 1

\begin{tabular}{ccccccccccc}
\hline Cucharas & Vaciadas & $\begin{array}{c}\text { Turno } \\
\text { anterior }\end{array}$ & \multicolumn{1}{c}{ Tiempos por cuchara (min) } & Suma & Promedio \\
\hline 4 & 5 & 54 & 45 & 74 & 78 & 97 & 294 & 73,5 \\
5 & 5 & 37 & 52 & 73 & 93 & 94 & 312 & 78,0 \\
6 & 5 & 24 & 54 & 61 & 86 & 21 & 222 & $55,5^{*}$ \\
7 & 6 & 19 & 62 & 51 & 61 & 43 & 133 & 350 & 70,0 \\
\hline Total & 21 & & & & & & & & \\
\hline
\end{tabular}

*Proceso con mayor eficiencia observada.

Elaboración propia

Tabla 14

Resultados del análisis del tiempo de vaciado del proceso de cucharas en el turno 2

\begin{tabular}{cccccccccc}
\hline Cuchara & Vaciadas & $\begin{array}{c}\text { Turno } \\
\text { anterior }\end{array}$ & \multicolumn{9}{c}{$\begin{array}{c}\text { Tiempos por cucharas } \\
\text { (min) }\end{array}$} & Suma & Promedio \\
\hline 4 & 5 & 45 & 48 & 74 & 60 & 42 & & 224 & 56,0 \\
5 & 6 & 20 & 77 & 46 & 85 & 63 & 56 & 327 & 65,4 \\
6 & 6 & 33 & 91 & 44 & 71 & 47 & 64 & 317 & 63,4 \\
7 & 5 & 49 & 41 & 72 & 53 & 56 & & 222 & $55,5^{\star}$ \\
\hline Total & 22 & & & & & & & &
\end{tabular}

* Proceso con mayor eficiencia observada.

Elaboración propia 
Tabla 15

Resultados del análisis del tiempo de vaciado del proceso de cucharas en el turno 3

\begin{tabular}{ccccccccccc}
\hline Cuchara & Vaciadas & $\begin{array}{c}\text { Turno } \\
\text { anterior }\end{array}$ & \multicolumn{1}{c}{ Tiempos por cucharas (min) } & Suma & Promedio \\
\hline 4 & 4 & 71 & 66 & 120 & 85 & & 271 & 90,33 \\
5 & 6 & 54 & 61 & 55 & 79 & 56 & 58 & 309 & $61,8^{*}$ \\
6 & 5 & 38 & 62 & 57 & 192 & 27 & 338 & 84,5 \\
7 & 5 & 18 & 45 & 57 & 169 & 77 & 348 & 87,00 \\
\hline Total & 20 & & & & & & & & &
\end{tabular}

* Proceso con mayor eficiencia observada.

Elaboración propia

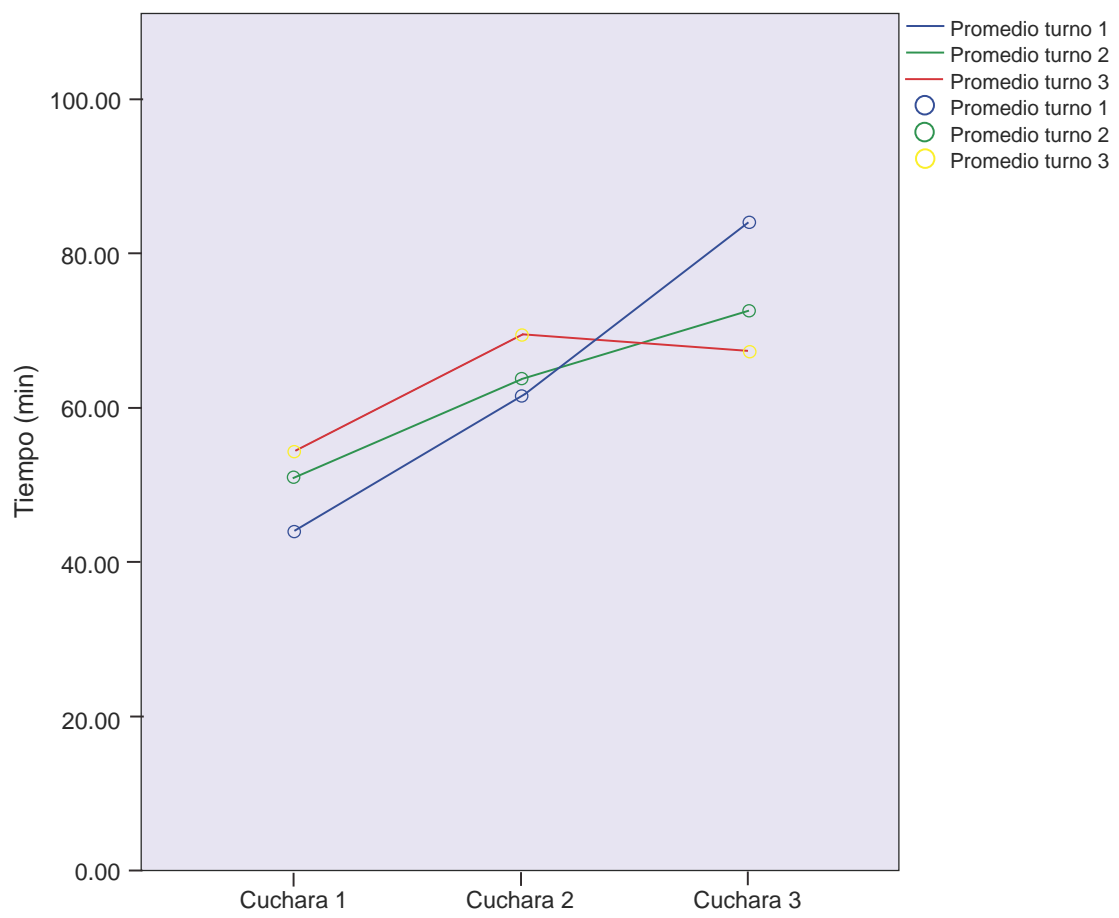

Figura 8. Comparación de los tiempos promedio de vaciado del proceso de cucharas del módulo 1

Elaboración propia 


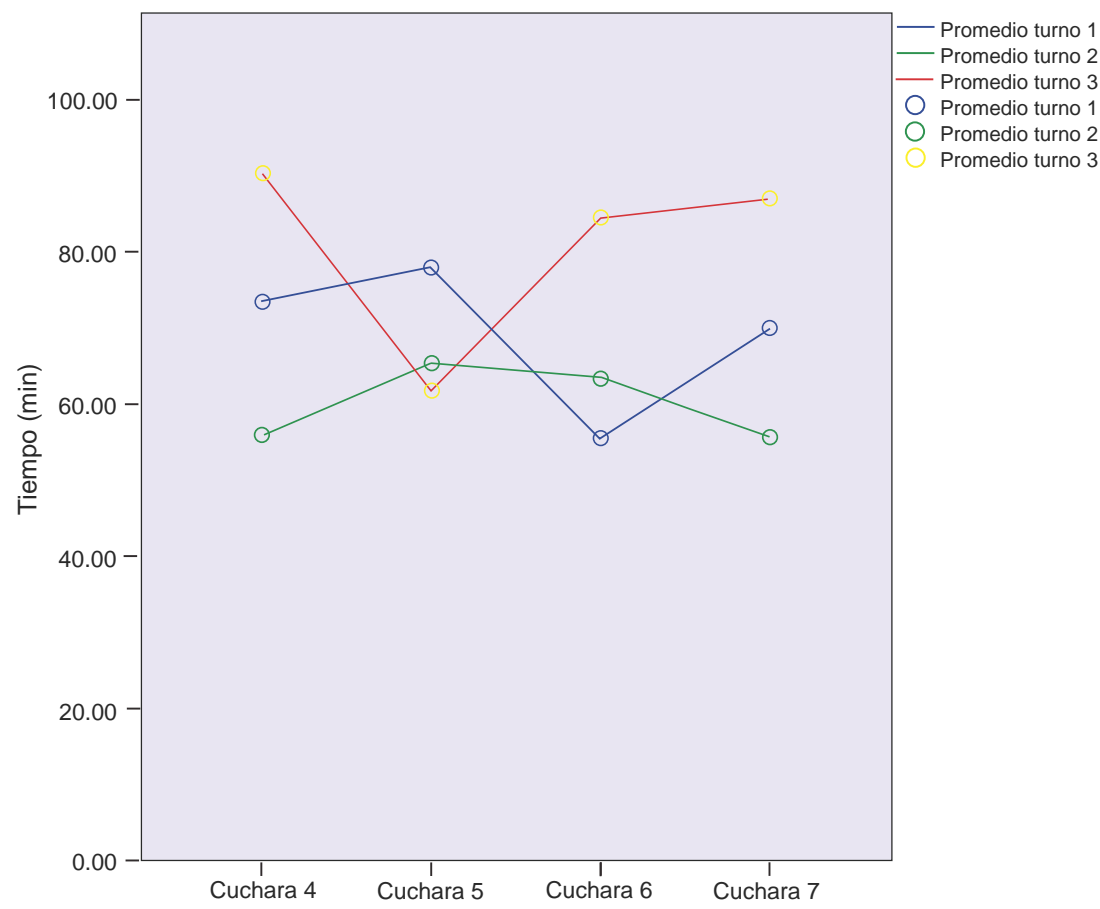

Figura 9. Comparación de los tiempos promedio de vaciado del proceso de cucharas del módulo 2

Elaboración propia

De acuerdo con el comparativo de turnos de la figura 8 referente al módulo 1, se aprecia que la cuchara 1 es la que presenta los tiempos promedio de proceso más bajos, con menor variabilidad entre los turnos $\mathrm{y}$, por lo tanto, la más eficiente en los tres turnos. Podemos notar que en este módulo la variabilidad que hay entre turnos en cada una de las cucharas es poca, y se aprecia también que la cuchara 3 es la menos eficiente.

$\mathrm{Al}$ respecto de la figura 9 referente al módulo 2, se observa que hay mayor variabilidad en la eficiencia de las cucharas en cada uno de los turnos; por ejemplo, tenemos que en el primer turno la más eficiente es la 3 ; en el segundo turno es la número 4; y en el tercer turno se presenta la número 2. En general, se observa un efecto de interacción entre los turnos y las cucharas en este módulo 2. 


\section{CONCLUSIONES}

Cada una de las observaciones y resultados son con el fin de poder tomar mejores decisiones en este tipo de procesos de sinterizado en compañías dedicadas al ramo minero y que a todas las empresas de este ramo que cuentan con este proceso les sirva de referencia para la mejora continua de sus sistemas.

De acuerdo con los resultados analizados en el proceso de sinterizado del proceso de cucharas específicamente, se llegó a las siguientes conclusiones:

1. El módulo más productivo es el 2 (54 \% de tiempo productivo directo), a diferencia del módulo 1 (53\% de tiempo productivo directo).

2. Lo anterior es debido a que tienen menores tiempos de espera y los tiempos de proceso en cucharas son similares (módulo 2), a diferencia del módulo 1, en el que los tiempos de proceso varían de cuchara a cuchara. No hay estandarización.

3. Falta de programa de mantenimiento preventivo en todas las áreas.

4. En los equipos pesados, en el mantenimiento programado (turno 2), se utilizan hasta dos horas, ya que se incluye la hora de comida.

Como parte de los resultados de este trabajo de investigación del proceso de cucharas, también se proponen recomendaciones de todo el proceso de sinterizado como aportación para poder generar una mejor toma de decisiones:

De acuerdo con el resultado del estudio y tomando en cuenta las condiciones actuales de la planta sinterizadora, en este momento no es posible prescindir de ninguno de los operadores y tampoco realizar contrataciones en dicha planta. Sin embargo, si se estandarizan las operaciones, se mejorarán las condiciones de los equipos con un buen programa de mantenimiento correctivo-preventivo y se implementarán dispositivos mecánicos en áreas que así lo requieran, y en un corto a mediano plazo se podrán realizar ajustes en el personal de esta área. 


\section{REFERENCIAS}

Beaverstock, M., Nordgren, B. y Greenwood, A. (2011). Applied Simulation. Modeling and Analysis Using Flexsim, Orem: Shanna Warr.

Campos, M., Blanco, L., Sicre-Artalejo, J. y Torralba, J. M. (2008). Aceros de baja aleación y alto rendimiento. Revista de Metalurgia, (44), 5-12.

DeGarmo, E. P., Black, J. T. y Kohser, R. A. (2003). Materials and Processes in Manufacturing. New York: John Wiley \& Sons.

Groover, P. M., Barrientos, M. A., León, C. J. y Reyes, R.R. (2007). Fundamentos de manufactura moderna. México: McGraw-Hill.

Kalpakjia, S. y Schmid, S. (2015). Manufactura, ingeniería y tecnología. México: Pearson.

Montes, J., Rodríguez, A., y Herrera, E. J. (2003). Consolidación de polvo de hierro mediante sinterización por resistencia eléctrica. Revista de Metalurgia, (39), 99-106.

Prado, J. M. y Riera, M. D. (1998). Simulación del proceso de compactación de polvos metálicos. Revista de Metalurgia, (34), 338-341.

Randall, M. (1989). Particle Packing Characteristics. Princeton, N. J.: Metal Powder Industries Federation.

Torralba, J. M. y Campos, M. (2014). Toward High Performance in Powder Metallurgy. Revista de Metalurgia, 50(2), pp. e017. doi: 10.3989/revmetalm.017 
Análisis de desempeño del área de cucharas en un proceso de sinterizado 